patients underwent OGDs pre-operatively and for what indication and with what result. Further sub-analysis was performed for each operation type.

Results 147 OGDs were done on a total of 116 bariatric surgical patients, with 23 patients having had more than one OGD each. Of these 147 OGDs 44 were pre-operative. 13 (29.5\%) OGD referrals were made to investigate anaemia, 12 (27.3\%) for pre-surgical screening to investigate existing symptoms of gastric ulceration, and $9(20.5 \%)$ to investigate abdominal pain. The remaining referrals were made for interventional gastric balloon insertions and removals $(6=13.6 \%)$ and to investigate symptoms of reflux $(3=6.8 \%)$ and dysphagia $(1=2.3 \%)$. The majority of patients $(50 \%)$ were referred prior to having a roux-en-y gastric bypass operation. Most of the pre-operative OGD findings were normal $(16=36.4 \%)$, but gastritis $(6=13.6 \%)$, hiatal hernias $(6=13.6 \%)$, gastric ulceration $(2=4.5 \%)$, oesophagitis $(1=2.3 \%)$ and duodenitis $(1=2.3 \%)$ were noted. Of the 13 patients referred with anaemia, $9(69.2 \%)$ had normal mucosa on OGD, but of the 12 patients who had pre-surgical screening 7 $(58.3 \%)$ were found to have abnormalities, including a fundic gland polyp and antral erosions.

Conclusion Using a selective referral process, only $4 \%$ of all bariatric surgery cases performed required preoperative endoscopy. The commonest indication for OGD preoperatively was anaemia and the commonest pathology found was mucosal inflammation (gastritis, oesophagitis and duodenitis).

Competing interests None declared.

\section{PMO-223 TATTOOING OF SUSPICIOUS COLONIC LESIONS AT COLONOSCOPY: IS ADHERENCE TO LOCAL PROTOCOL BETTER IF IDENTIFIED THROUGH THE BOWEL CANCER SCREENING PROGRAMME?}

doi:10.1136/gutjnl-2012-302514b.223

${ }^{1} \mathrm{H}$ Travers, ${ }^{*}{ }^{2} \mathrm{~T}$ Shirazi, ${ }^{1} \mathrm{~S}$ Mansfield. ${ }^{1}$ Department of Colorectal Surgery, Royal Devon and Exeter Hospital, Exeter, UK; ${ }^{2}$ Department of Gastroenterology, Royal Devon and Exeter Hospital, Exeter, UK

Introduction The Joint Advisory Group on gastrointestinal endoscopy and the National Bowel Cancer Screening Programme (BCSP) have published guidelines on the tattooing of malignant and suspicious lesions at colonoscopy. Our endoscopy department has a local protocol for the tattooing of these lesions based on these guidelines. The BCSP has led to an increase in the number of colonoscopies performed and the number of lesions identified.

Aim To assess adherence to a local protocol in a single endoscopy unit and identify if lesions identified through the BCSP are more likely to be tattooed than lesions identified for other reasons.

Methods A retrospective review of a prospectively maintained database was performed. All colonoscopies performed between 1 April 2010 and 31 March 2011 were reviewed and screening cases identified.

Results 4023 colonoscopies were performed, 307 (8\%) as part of the BCSP. Malignancy or polyps were identified in 192 (63\%) of BSCP colonoscopies compared to $26 \%$ (958/3716) of non-BCSP colonoscopies. Significantly more polyps and malignancies were identified during BCSP colonoscopies than non-BCSP colonoscopies $(p<0.0001$ $\chi^{2}$ test). Our local protocol states that any malignant/suspicious/ $>1 \mathrm{~cm}$ lesion distal to the right colon should be marked by placing three tattoos just distal to the lesion. 94 (49\%) lesions were identified during BCSP colonoscopies that met these criteria. Of these 54 $(57 \%)$ were tattooed, and $20(21 \%)$ were tattooed by the method advised. This compared to 262 non-BCSP lesions identified that should have been tattooed of which 77 (29\%) were tattooed and 20 (8\%) were tattooed by the method advised. Tattooing rate was significantly higher in BCSP detected lesions (54/94 compared with $77 / 262, \mathrm{p} \leq 0.0001, \chi^{2}$ test).
Conclusion Tattooing practice in our endoscopy unit is poor despite the presence of a local protocol. However, tattooing practice is significantly better in lesions identified through the BCSP. Reasons for this may include the higher yield of lesions in screening colonoscopies or lack of awareness of the protocol. We aim to improve adherence by increasing awareness among all endoscopy staff to ensure optimum management of malignant and suspicious lesions.

Competing interests None declared.

\section{REFERENCES}

1. Chilton A, Rutter $\mathrm{M}$, et al. Quality assurance guidelines for colonoscopy. NHS BCSP Publication 6. 2010.

2. BSG Quality and Safety indicators for Endoscopy, Joint Advisory Group on GI Endoscopy. 2007. http://www.thejag.org.uk/downloads\%5CUnit\%20Resources\% 5CBSG\%200uality\%20and\%20Safety\%20Indicators.pdf

\section{Inflammatory bowel disease I

PMO-224 NF-KB2 DELETION PROTECTS MURINE COLON AGAINST
DSS-INDUCED COLITIS AND THIS IS ASSOCIATED WITH
REDUCED EXPRESSION OF TNF- $\alpha$ AND IL14

doi:10.1136/gutjnl-2012-302514b.224

${ }^{1} \mathrm{~A}$ Hanedi, ${ }^{1} \mathrm{M}$ D Burkitt, ${ }^{1} \mathrm{C}$ A Duckworth, ${ }^{2} \mathrm{R}$ Dimaline, ${ }^{3} \mathrm{~J} \mathrm{H}$ Caamano, ${ }^{1} \mathrm{D}$ M Pritchard. 'Gastroenterology, University of Liverpool, Liverpool, UK; ${ }^{2}$ Physiology, University of Liverpool, Liverpool, UK; ${ }^{3}$ BBR-MRC Centre for Immune Regulation, University of Birmingham, Birmingham, UK

Introduction The Nuclear Factor kappa B (NFKB) family of five transcription factors signals via two pathways (classical and alternative). Classical pathway NFKB signalling has previously been implicated in the pathogenesis of inflammatory bowel diseases (IBD). However, the role of alternative NFKB pathway activation in the development of intestinal inflammation has not previously been investigated.

Aims To investigate the susceptibility of mice deficient in two individual NFKB family members to DSS-induced colitis and the associated molecular changes.

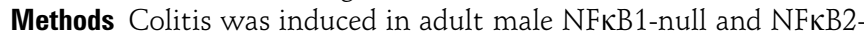
null mice and their wild-type (C57BL/6) counterparts by oral $2 \%$ DSS administration for 5 days ( $n=10$ per group). Weight loss and Disease Activity Index (DAI) were evaluated daily. Animals were euthanased on day 6 and histological colitis severity was evaluated in $\mathrm{H} / \mathrm{E}$ stained colonic sections. The colonic expression of 6 key proinflammatory cytokines (TNF- $\alpha$, IL- $1 \beta$, INF- $\gamma$, IL- 6 and IL-14) was assessed by real time PCR ( $\mathrm{n}=4$ per group). Statistical comparisons were mostly performed by ANOVA with Bonferroni post-hoc tests, but the Kruskal-Wallis with Dunn's multiple comparison test was used to analyse DAI and histological scores.

Results After oral administration of $2 \%$ DSS, NFKB1-null mice showed significantly more loss of body weight whereas NFKB2-null mice showed significantly less loss of body weight on days 5 and 6 compared to wild-type mice. DAI was also significantly higher in

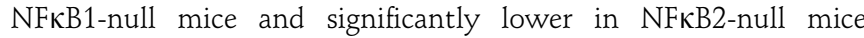
compared to $\mathrm{C} 57 \mathrm{BL} / 6$ mice. In agreement with these clinical findings, histological assessment of DSS treated animals confirmed a severely damaged and inflamed distal colon in C57BL/6 and NFKB1null mice and minimal histological damage and significantly lower inflammation scores in NFKB2-null mice. The expression of IL-6 mRNA was significantly increased in DSS-treated NFKB1-null colon and the expressions of TNF- $\alpha$ and IL-14 mRNAs were significantly reduced in DSS-treated NFKB2-null colon

Conclusion Disruption of the classical NFKB signalling pathway by deleting NFKB1 exacerbates colonic inflammation and tissue damage following DSS administration which may be partially mediated by IL-6. This suggests that classical NFKB pathway inhibitors may be 
paradoxically harmful in IBD. However, disruption of the alternative pathway by deleting NFKB2 protected murine colon from developing inflammation and this was associated with reduced expression of TNF- $\alpha$ and IL-14. Pharmacological inhibition of the NFKB2 signalling pathway may therefore be a promising novel therapeutic strategy for IBD.

Competing interests None declared.

\section{PMO-225 USE OF INFLIXIMAB FOR ACUTE SEVERE ULCERATIVE COLITIS IN A DISTRICT GENERAL HOSPITAL}

doi:10.1136/gutjnl-2012-302514b.225

A Alisa,* S Musa, S Zar, A Mahmood. Gastroenterology, St Helier Hospital, London, UK

Introduction The prevalence of Ulcerative colitis (UC) in the UK is $243 / 100000$ and carries a high lifetime risk of surgery $(20 \%-30 \%)$. Acute severe UC (ASUC) is potentially a life threatening condition. Traditionally urgent medical treatment includes intravenous steroids followed by IV Ciclosporin in non-responders. In 2010, NICE recommended the use of Infliximab in ASUC in patients in whom ciclosporin is contraindicated or clinically inappropriate. Randomised control trials suggest colectomy is required in 30\% of Infliximab recipients at 3 months and $50 \%$ at 3 years. ${ }^{12}$ This retrospective study reports our experience at a busy district general hospital in South West London.

Methods The Biologic database at St. Helier's Hospital was interrogated to identify all cases of ASUC that gained funding for. Infliximab data collection was from January 2009 to June 2011. All patients had a UC disease activity index score (UCDAI) $>10$. Disease site was classified by the Montreal classification. Follow-up ranged from 6 to 36 months post treatment.

Results 20 patients had funding approved. 17/20 (85\%) patients received Infliximab, 3/20 (15\%) clinically settled pre-administration. There were $9(53 \%)$ males and 8 (47\%) females, median age 47 (range 19-57). 9 (53\%) patients had left sided disease and $8 / 17$ (47\%) had extensive colitis. Median UCDAI was 11 (range 10-13). The median disease duration 4 years (range $0-15$ years). During the acute presentation (pre-Infliximab), 11/17 (65\%) patients received steroids, $11 / 17$ (65\%) were already on azathioprine, 15/17 (88\%) 5-ASA, 3/17 (18\%) 6-MP and 1/17 (6\%) Tacrolimus. Following steroid therapy 4/ $17(24 \%)$ had received Ciclosporin. Urgent colectomy was required in $2 / 17$ (12\%) patients. A further 6/17 (35\%) underwent elective colectomy post induction, median 5 months (range $2-12$ months). Complications were experienced in 3/17 (18\%) and included 1 lichen planus, 1 Raynauds, 1 arthralgia. There was no mortality reported. Conclusion Following Infliximab therapy urgent colectomy was avoided in the majority ( $82 \%$ ) of patients. Our elective colectomy rate was not to dissimilar to those reported by main randomised control trials. Infliximab was well tolerated and provided rescue therapy prior to latent elective surgery.

Competing interests None declared.

\section{REFERENCES}

1. Jarnerot G, et al. Gastroenterology 2005;128:1805-11.

2. Gustavsson A, et al. Aliment Pharmacol Ther 2010;32:984-9.

\section{PM0-226 DIFFERING PHENOTYPE IN ELDERLY IBD; SHOULD MONTREAL INCLUDE AN A4 CATEGORY}

doi:10.1136/gutjnl-2012-302514b.226

A A McNicol, ${ }^{*}$ N Kennedy, J Satsangi, I D Arnott. ${ }^{1}$ GI Unit, Western General Hospital, Edinburgh, UK

Introduction The phenotype of elderly-onset IBD is poorly described and knowledge lags behind that of other age-groups. While not the dominant age-group in the disease population, those diagnosed over 60 will compose a larger proportion it as the general population ages over the next decade. The Montreal classification for IBD stratifies related to age into three categories, 40(A3) the study aims to ascertain if disease phenotype varies between those aged 40-59 and those $>60$ with possibility of an A4 group becoming viable if variance is noted.

Methods 1957 patients with IBD were identified using the IBD database at the Western General Hospital. We selected all those UC $(n=306)$ and CD $(n=135)$ diagnosed at age 40 and over (A3) and subdivided the group in to those over and under 60 . The diagnosis adhered to the criteria of Lennard-Jones and IBD was categorised according to the Montréal classification. Data collected included diagnosis, age at diagnosis, disease distribution, disease behaviour and smoking history. Follow-up were available for 5 years following diagnosis. Analysis of the groups was undertaken using $\chi^{2}$ and Fishers exact test.

Results Gender of CD patients in the different age groups (40-59; $\mathrm{M} / \mathrm{F}=53 / 96 . \quad>60 ; \mathrm{M} / \mathrm{F}=10 / 49 \mathrm{p}=0.0115)$ illustrated a higher proportion of women in the $>60$ group. CD patients who were diagnosed over the age of 60 had more isolated colonic disease at diagnosis. (L2; 40-59 N=28/77, >60 N=37/58, $\mathrm{p}=0.0032 \mathrm{~L} 3 ; 40-59$ $\mathrm{N}=15 / 77,>60 \mathrm{~N}=2 / 58 \mathrm{p}=0.0073)$. By 5 years of follow-up these differences were no longer significant. There was do difference in disease behaviour or smoking history. UC patients had more left sided disease and less distal disease at diagnosis (E1; 40-59 $\mathrm{N}=70$ / 204, $>60 \mathrm{~N}=20 / 102, p=0.0079 \mathrm{E} 2 ; 40-59 \mathrm{~N}=88 / 204,>60 \mathrm{~N}=57 /$ $102 \mathrm{p}=0.039)$. Smoking history showed a greater proportion of former smokers in the $>60$ group $(40-59 \mathrm{~N}=108 / 216,>60 \mathrm{~N}=70 /$ $107 \mathrm{p}=0.0058$ ).

Conclusion Disease phenotype at Dx in both UC and Crohn's differs in the over-60s at diagnosis but normalises to that of the $A 3$ population at follow-up. This data suggests that the introduction of an additional Montreal age classification, A4, would be clinically meaningful. Further analysis will demonstrate whether response to treatment differs in this age group.

Competing interests None declared.

\section{PM0-227 INFLIXIMAB INDUCTION THERAPY ALONE FOR ULCERATIVE COLITIS DOES NOT RESULT IN LONG TERM REMISSION}

doi:10.1136/gutjnl-2012-302514b.227

A J Brooks, ${ }^{*}$ K Robinson, A Wright, M E McAlindon, A Lobo. Gastroenterology, Sheffield Teaching Hospitals NHS Foundation Trust, Sheffield, UK

Introduction Infliximab (IFX) has demonstrated efficacy in moderate to severe ulcerative colitis (UC) with a reduction in short-term colectomy rates. ${ }^{1}$ In the UK, the National Institute for Health and Clinical Excellence (NICE) guidance relates to an induction course of three-doses for severely active ulcerative colitis. ${ }^{2}$ The aim of this study was to determine outcomes following IFX induction, including colectomy rate, use of corticosteroids (CS) or repeat IFX induction.

Methods Patients with UC at a single large teaching centre received IFX induction for UC requiring hospitalisation or when urgent consideration of surgery was given for resistant or rapidly relapsing disease were retrospectively reviewed (2008-2011). All patients had a Simple Colitis Activity Index (SCAI) at 0, 2, 6 weeks.

Results Twenty-seven patients were studied, median age 38 (range 23-64), with 17 (63\%) refractory to oral or intravenous CS (13 and 4 respectively). All received CS in the year preceding IFX; median 1 course (range 1-4). 23 (85\%) were on immunosuppression (IS) (16 thiopurines, 7 methotrexate), 3 intolerant or non-responsive and 1 naïve to IS. Twenty (74\%) received induction IFX alone. Median SCAI was 8 (range 4-13), 4 (range 0-9), 2 (range $0-10$ ) at 0,2 and 